\title{
Mean-Variance-Entropy Portfolio Selection Models with Uncertain Returns
}

\author{
Shengguo Li ${ }^{1, *}$, Jin Peng ${ }^{1}$, Bo Zhang ${ }^{2}$, Dan Ralescu ${ }^{3}$ \\ ${ }^{1}$ Institute of Uncertain Systems, Huanggang Normal University, Huanggang, China \\ ${ }^{2}$ School of Statistics and Mathematics, Zhongnan University of Economics and Law, Wuhan, China \\ ${ }^{3}$ Department of Mathematical Sciences, University of Cincinnati, Cincinnati, USA
}

\section{Email address:}

lisg@hgnu.edu.cn (Shengguo Li)

${ }^{*}$ Corresponding author

\section{To cite this article:}

Shengguo Li, Jin Peng, Bo Zhang, Dan Ralescu. Mean-Variance-Entropy Portfolio Selection Models with Uncertain Returns. International Journal of Management and Fuzzy Systems. Vol. 7, No. 3, 2021, pp. 47-54. doi: 10.11648/j.ijmfs.20210703.12

Received: June 30, 2021; Accepted: July 16, 2021; Published: August 23, 2021

\begin{abstract}
Diversification portfolio selection problem is an important issue in uncertain economic environment. In this paper, this problem is discussed within the framework of uncertainty theory. First, an uncertain extension mean-variance diversification model is proposed, in which the mean is chosen as the objective function, and variance and proportion entropy as risk and diversity constraints. Then two variations are investigated on the purposes of minimizing the risk and maximizing the diversity measure, respectively. Furthermore, the corresponding analytical mathematical models are deduced via the convenient operational law of uncertain variables. Finally, several numerical examples are given to illustrate the modeling idea. The results showed that the diversification models had higher diversification than the uncertain mean-variance model. The proposed models provide a new method to make decision-making in uncertain portfolio selection problem.
\end{abstract}

Keywords: Portfolio Selection, Uncertain Variable, Entropy, Uncertainty Modeling

\section{Introduction}

Portfolio selection discusses the problem of how to allocate one's capital to a large number of securities so that the investment can bring a most profitable return. In 1952, Markowitz [8] initialized the portfolio selection problems by mean-variance methodology. After that, many extensions to the mean-variance model have been proposed [16, 22]. As is well known, the variance is a common and useful risk measure in portfolio selection models. However, it is only an average deviation measure of information. In order to solve this problem, it is necessary to introduce a measure of diversification to portfolio selection models. Inspired by the Shannon entropy [6], for investment proportions in the securities, some researchers introduced the proportions entropy. It is an effective tool as the diversification measure. Golan et al. [2] used the entropy itself as the objective function to obtain maximum diversification. Bera and Park [1] chose the cross entropy measure as the objective function to investigate the optimal portfolio diversification through the maximum entropy approach. Usta and Kantar [9] and Jana et al. [15] added entropy as one of the objectives to discuss the the multi-objective models and generated the well diversification portfolio. Huang [18] used mean as the objective function and proposed the diversification models via random entropy constraint. In addition, Kapur and Kesavan [11], Fang et al. [17] successively improved the traditional mean-variance models with the help of the entropy.

In the above portfolio selection, a randomness label was put on the security returns and the security returns were regarded as random variables. Obviously, the investor should have enough historical data when using probability theory. However, for various reasons not all returns data are observed. For example, for the security return of a new type of stock, it is impossible to obtain a large number of historical data about it. Thus it is difficult to estimate the probability distribution of the security return. In order to deal with such problems, the domain experts are invited to evaluate the belief degrees about it. Then this may lead to a counterintuitive result if to model 
these belief degrees by probability.

Next, a numerical example is provided to explain it. Within a month, assume the returns of every day from this new type of stock are iid uniform random variables on $[0,10]$ in dollar terms. Obviously, it is easy to verify that

$$
\operatorname{Pr}\{\text { "the return of every day is less than } 10 \text { dollars in a month" }\}=1 .
$$

That is, we are $100 \%$ sure that the returns are less than 300 dollars within a month.

However, when there are no samples for the return, some insurance experts are invited to evaluate the belief degrees about it. It follows from the view of Kahneman and Tversky [7] that the insurance experts usually provide a much wider range of return than the actual return. Assume the belief degree

$$
\operatorname{Pr}\{\text { "the return of every day is less than } 10 \text { dollars" }\}=0.67 \text {. }
$$

And

$$
\operatorname{Pr}\{\text { "the return of every day is less than } 10 \text { dollars in a month" }\}=0.67^{30} \approx 0 \text {. }
$$

That is, it is almost impossible that the returns are less than 300 dollars within a month. The opposite results (1) and (3) show that a sure event becomes an impossible one. It seems unacceptable. So the belief degrees cannot be regarded as probability.
In order to deal with the above problem, Liu [3] founded uncertainty theory. If the belief degree function is treated as a linear uncertainty distribution on $[0,15]$ in dollars (See Figure 1), then

$$
\mathrm{M}\{\text { "the return of every day is less than } 10 \text { dollars in a month" }\}=\underbrace{0.67 \wedge 0.67 \wedge \cdots \wedge 0.67}_{30}=0.67 \text {. }
$$

That is, the returns less than 300 dollars within a month is $67 \%$. Although the degree $67 \%$ is smaller than the true value $100 \%$, this is a more realistic result.

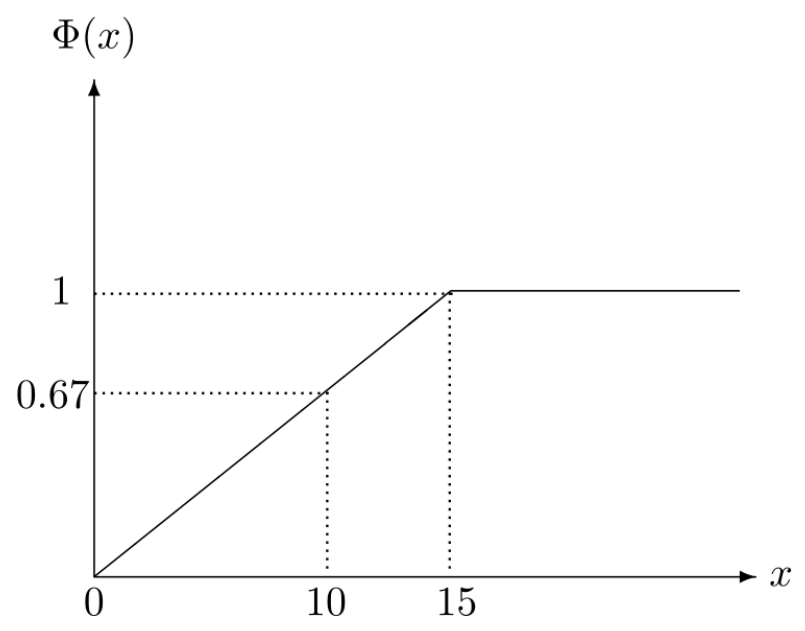

Figure 1. Belief degree function of "the daily return".

As a branch of mathematics to cope with the uncertainty in human systems, uncertainty theory provides a new approach to study the portfolio selection problems. Based on the assumption that security returns are uncertain variables, Yan
[14] proposed an uncertain mean-variance model in which the variance was a risk measure. Zhang et al. [5] built an uncertain expected-variance-chance portfolio selection model, where the objective was to maximise the result of expected value of the portfolio divided by variance. Ning et al. [21] discussed an uncertain mean-variance model with an uncertain triangular entropy constraint. Chen et al. [13] proposed the semivariance concept of uncertain variables, and gave meansemivariance model. Chang et al. [10] discussed an uncertain multi-period portfolio selection problem with mental accounts and realistic constraints. Here the entropy will be introduced as the diversification measure into uncertain mean-variance portfolio selection model and build uncertain diversification portfolio selection models.

The remainder of this paper is organized as follows. In Section 2, some concepts and properties from uncertainty theory are recalled. The concept of Shannon entropy and its properties are recalled in Section 3. Some uncertain diversification models for portfolio selection are proposed in Section 4. Several numerical examples for understanding the new models are given in Section 5. The last section contains a brief summary. 


\section{Preliminary}

Let $\Gamma$ be a nonempty set and $\mathcal{L}$ a $\sigma$-algebra over $\Gamma$. Each element $\Lambda \in \mathcal{L}$ is assigned a number $M\{\Lambda\}$. The set function $M$ is called by Liu [3] an uncertain measure if it satisfies the normality, duality, and subadditivity axioms. The triplet $(\Gamma, \mathcal{L}, M)$ is called an uncertainty space.

Definition 2.1. (Liu [3]) An uncertain variable is defined as a measurable function from an uncertainty space $(\Gamma, \mathcal{L}, M)$ to the set of real numbers, i.e., for any Borel set $B$ of real numbers, the set

$$
\{\xi \in B\}=\{\gamma \in \Gamma \mid \xi(\gamma) \in B\}
$$

is an event.

Definition 2.2. (Liu [3]) The uncertainty distribution $\Phi$ : $\Re \rightarrow[0,1]$ of an uncertain variable $\xi$ is defined by

$$
\Phi(x)=M\{\gamma \in \Gamma \mid \xi(\gamma) \leq x\}
$$

for every real number $x$.

Peng and Iwamura [23] have proved that a function is an uncertainty distribution function if and only if it is an increasing function except $\Phi(x) \equiv 0$ and $\Phi(x) \equiv 1$.

Definition 2.3. (Liu [3]) Let $\xi$ be an uncertain variable. Then the expected value of uncertain variable $\xi$ is defined by

$$
E[\xi]=\int_{0}^{+\infty} M\{\xi \geq r\} \mathrm{d} r-\int_{-\infty}^{0} M\{\xi \leq r\} \mathrm{d} r
$$

provided that at least one of the two integrals is finite.

Let $\xi$ be an uncertain variable with finite expected value $E[\xi]$. The variance of $\xi$ is defined as $V[\xi]=E\left[(\xi-E[\xi])^{2}\right]$. The standard deviation of $\xi$ is defined as $\sqrt{V[\xi]}$.
An uncertain variable $\xi$ is called normal if it has a normal uncertainty distribution

$$
\Phi(x)=\left(1+\exp \left(\frac{\pi(e-x)}{\sqrt{3} \sigma}\right)\right)^{-1}, x \in \Re,
$$

denoted by $\mathcal{N}(e, \sigma)$, where $e$ and $\sigma$ are real numbers with $\sigma>0$. The expected value and variance are $E[\xi]=e$ and $V[\xi]=\sigma^{2}$, respectively.

Definition 2.4. (Liu [3]) An uncertainty distribution $\Phi$ is said to be regular if its inverse function $\Phi^{-1}(\alpha)$ exists and is unique for each $\alpha \in(0,1)$.

It follows from Definition 2.4 that a normal uncertainty distribution is regular and its inverse uncertainty distribution is

$$
\Phi^{-1}(\alpha)=e+\frac{\sqrt{3} \sigma}{\pi} \ln \frac{\alpha}{1-\alpha}, \quad(0<\alpha<1) .
$$

Theorem 2.1. (Liu [4]) Let $\xi$ be an uncertain variable with regular uncertainty distribution $\Phi$. If the expected value exists, then

$$
E[\xi]=\int_{0}^{1} \Phi^{-1}(\alpha) \mathrm{d} \alpha .
$$

In order to calculate the uncertainty distribution of monotone functions of uncertain variables, the operational law of independent uncertain variables was proved by Liu [4].

Theorem 2.2. (Liu [4]) Let $\xi_{1}, \xi_{2}, \cdots, \xi_{n}$ be independent uncertain variables with regular uncertainty distributions $\Phi_{1}, \Phi_{2}, \cdots, \Phi_{n}$, respectively. If $f\left(x_{1}, x_{2}, \cdots, x_{n}\right)$ is a strictly increasing function with respect to $x_{1}, x_{2}, \cdots, x_{m}$ and strictly decreasing with respect to $x_{m+1}, x_{m+2}, \cdots, x_{n}$, then $\xi=f\left(\xi_{1}, \xi_{2}, \cdots, \xi_{n}\right)$ is an uncertain variable with inverse uncertainty distribution

$$
\Psi^{-1}(\alpha)=f\left(\Phi_{1}^{-1}(\alpha), \cdots, \Phi_{m}^{-1}(\alpha), \Phi_{m+1}^{-1}(1-\alpha), \cdots, \Phi_{n}^{-1}(1-\alpha)\right)
$$

Liu and Ha [20] proved the following formula of the expected value of functions of uncertain variables.

Theorem 2.3. (Liu and $\mathrm{Ha}$ [20]) Let $\xi_{1}, \xi_{2}, \cdots, \xi_{n}$ be independent uncertain variables with regular uncertainty distributions $\Phi_{1}, \Phi_{2}, \cdots, \Phi_{n}$, respectively. If $f\left(x_{1}, x_{2}, \cdots, x_{n}\right)$ is a strictly increasing function with respect to $x_{1}, x_{2}, \cdots, x_{m}$ and strictly decreasing with respect to $x_{m+1}, x_{m+2}, \cdots, x_{n}$, then the uncertain variable $\xi=$ $f\left(\xi_{1}, \xi_{2}, \cdots, \xi_{n}\right)$ has an expected value

$$
E[\xi]=\int_{0}^{1} f\left(\Phi_{1}^{-1}(\alpha), \cdots, \Phi_{m}^{-1}(\alpha), \Phi_{m+1}^{-1}(1-\alpha), \cdots, \Phi_{n}^{-1}(1-\alpha)\right) \mathrm{d} \alpha
$$

Provided that $E[\xi]$ exists.

Recently, Yao [12] proved a formula to calculate the variance of an uncertain variable.

Theorem 2.4. (Yao [12]) Let $\xi_{1}, \xi_{2}, \cdots, \xi_{n}$ be independent uncertain variables with regular uncertainty distributions
$\Phi_{1}, \Phi_{2}, \cdots, \Phi_{n}$, respectively. If $f\left(x_{1}, x_{2}, \cdots, x_{n}\right)$ is a strictly increasing function with respect to $x_{1}, x_{2}, \cdots, x_{m}$ and strictly decreasing with respect to $x_{m+1}, x_{m+2}, \cdots, x_{n}$, then the uncertain variable $\xi=f\left(\xi_{1}, \xi_{2}, \cdots, \xi_{n}\right)$ has a variance

$$
V[\xi]=\int_{0}^{1} f\left(\Phi_{1}^{-1}(\alpha), \cdots, \Phi_{m}^{-1}(\alpha), \Phi_{m+1}^{-1}(1-\alpha), \cdots, \Phi_{n}^{-1}(1-\alpha)\right)^{2} \mathrm{~d} \alpha-e^{2}
$$


where $e$ is the expected value of $\xi=f\left(\xi_{1}, \xi_{2}, \cdots, \xi_{n}\right)$, i.e.,

$$
e=\int_{0}^{1} f\left(\Phi_{1}^{-1}(\alpha), \cdots, \Phi_{m}^{-1}(\alpha), \Phi_{m+1}^{-1}(1-\alpha), \cdots, \Phi_{n}^{-1}(1-\alpha)\right) \mathrm{d} \alpha .
$$

\section{Shannon Entropy}

Let $P$ be a partition of unity, i.e., $P$ is a collection of finitely many nonnegative numbers $\left\{p_{1}, p_{2}, \cdots, p_{n}\right\}$ with $\sum_{i=1}^{n} p_{i}=1$. Then the Shannon entropy of $P$ is defined by

$$
H[P]=-\sum_{i=1}^{n} p_{i} \ln p_{i}
$$

Obviously, the Shannon entropy represents the average amount of information gained by observing the partition, and it has some properties a follows:

(a) The entropy of a partition does not depend on the order in which the elements of the partition are numbered.

(b) The entropy of a partition is nonnegative and equals zero if and only if there exists a value $p_{i} \in\left\{p_{1}, p_{2}, \cdots, p_{n}\right\}$ such that $p_{i}=1$ (and all other are 0 ).

(c) The entropy of a partition is maximum $\ln n$ when $p_{1}=$ $p_{2}=\cdots=p_{n}=1 / n$.

Let $x_{1}, x_{2}, \cdots, x_{n}$ be the investment proportions in the securities. Then we have $x_{i} \geq 0, i=1,2, \cdots, n$ and $\sum_{i=1}^{n} x_{i}=1$.

Definition 3.1. Let $x_{1}, x_{2}, \cdots, x_{n}$ be the investment proportions in the securities. Then the proportion entropy is defined by

$$
H=-\sum_{i=1}^{n} x_{i} \ln x_{i} .
$$

It follows from the properties of Shannon entropy and Definition 3.1 that the greater the value of the proportion entropy, the more diversely the capital is allocated to the alternative securities. Next, we will propose the uncertain diversification models with uncertain returns proportion entropy.

\section{Uncertain Diversification Models}

Assume that the portfolio is $x_{1} \xi_{1}+x_{2} \xi_{2}+\cdots+x_{n} \xi_{n}$, where $\xi_{1}, \xi_{2}, \cdots, \xi_{n}$ are the independent uncertain return rates and $x_{1}, x_{2}, \cdots, x_{n}$ are the corresponding investment proportions. The return and the risk of the portfolio $x_{1} \xi_{1}+x_{2} \xi_{2}+\cdots+x_{n} \xi_{n}$ are quantified as the mean and variance, respectively. Let $\beta$ be the maximum risk level and $\gamma$ be the diversity measure level.
Then we have the following model

$$
\left\{\begin{array}{l}
\max E\left[x_{1} \xi_{1}+x_{2} \xi_{2}+\cdots+x_{n} \xi_{n}\right] \\
\text { subject to: } \\
V\left[x_{1} \xi_{1}+x_{2} \xi_{2}+\cdots+x_{n} \xi_{n}\right] \leq \beta \\
-\sum_{i=1}^{n} x_{i} \ln x_{i} \geq \gamma \\
x_{1}+x_{2}+\cdots+x_{n}=1 \\
x_{i} \geq 0, i=1,2, \cdots, n .
\end{array}\right.
$$

In this model, the objective is to maximize the return at the desired levels of risk and diversity. This model is an extension of the uncertain mean-variance model. If the second constraint does not exist, then the above model degenerates to the uncertain mean-variance model proposed by Yan [14].

In portfolio problems, another common objective is to minimize investment risk subject to the given expected return $\delta$ and level measure of diversity $\gamma$. This leads to the first variation of model (7) as follows,

$$
\left\{\begin{array}{l}
\min V\left[x_{1} \xi_{1}+x_{2} \xi_{2}+\cdots+x_{n} \xi_{n}\right] \\
\text { subject to: } \\
E\left[x_{1} \xi_{1}+x_{2} \xi_{2}+\cdots+x_{n} \xi_{n}\right] \geq \delta \\
-\sum_{i=1}^{n} x_{i} \ln x_{i} \geq \gamma \\
x_{1}+x_{2}+\cdots+x_{n}=1 \\
x_{i} \geq 0, i=1,2, \cdots, n .
\end{array}\right.
$$

Sometimes, the aim of the investor is to maximize the level measure of diversity when expected return is no less than some given target values $\delta$ and the variance level no more than $\beta$, respectively. Then we obtain the second variation of model (7)

$$
\left\{\begin{array}{l}
\max -\sum_{i=1}^{n} x_{i} \ln x_{i} \\
\text { subject to: } \\
E\left[x_{1} \xi_{1}+x_{2} \xi_{2}+\cdots+x_{n} \xi_{n}\right] \geq \delta \\
V\left[x_{1} \xi_{1}+x_{2} \xi_{2}+\cdots+x_{n} \xi_{n}\right] \leq \beta \\
x_{1}+x_{2}+\cdots+x_{n}=1 \\
x_{i} \geq 0, i=1,2, \cdots, n .
\end{array}\right.
$$

Theorem 4.1. Let $\xi_{1}, \xi_{2}, \cdots, \xi_{n}$ be independent uncertain variables with regular uncertainty distributions $\Phi_{1}, \Phi_{2}, \cdots, \Phi_{n}$, respectively. Then the model (7) can be converted into the following deterministic model, 


$$
\left\{\begin{array}{l}
\max \int_{0}^{1} \sum_{i=1}^{n} x_{i} \Phi_{i}^{-1}(\alpha) \mathrm{d} \alpha \\
\text { subject to: } \\
\int_{0}^{1}\left(\sum_{i=1}^{n} x_{i} \Phi_{i}^{-1}(\alpha)\right)^{2} \mathrm{~d} \alpha-\left(\int_{0}^{1} \sum_{i=1}^{n} x_{i} \Phi_{i}^{-1}(\alpha) \mathrm{d} \alpha\right)^{2} \leq \beta \\
-\sum_{i=1}^{n} x_{i} \ln x_{i} \geq \gamma \\
x_{1}+x_{2}+\cdots+x_{n}=1 \\
x_{i} \geq 0, i=1,2, \cdots, n .
\end{array}\right.
$$

Proof. Since $f\left(y_{1}, y_{2}, \cdots, y_{n}\right)=x_{1} y_{1}+x_{2} y_{2}+\cdots+x_{n} y_{n}$ is strictly increasing with respect to $y_{1}, y_{2}, \cdots, y_{n}$, it follows from Theorem 2.2 that the inverse uncertainty distribution of $\sum_{i=1}^{n} x_{i} \xi_{i}$ is

$$
\Psi^{-1}(\alpha)=\sum_{i=1}^{n} x_{i} \Phi_{i}^{-1}(\alpha)
$$

By using Theorem 2.3 and Theorem 2.4, we obtain

$$
E\left[\sum_{i=1}^{n} x_{i} \xi_{i}\right]=\int_{0}^{1} \sum_{i=1}^{n} x_{i} \Phi_{i}^{-1}(\alpha) \mathrm{d} \alpha
$$

and

$$
V\left[\sum_{i=1}^{n} x_{i} \xi_{i}\right]=\int_{0}^{1}\left(\sum_{i=1}^{n} x_{i} \Phi_{i}^{-1}(\alpha)\right)^{2} \mathrm{~d} \alpha-\left(E\left[\sum_{i=1}^{n} x_{i} \xi_{i}\right]\right)^{2} .
$$

Substituting these equations into model $(7)$, the theorem is proved.

Similarly, when security returns are all independent uncertain variables, model (8) is converted into the following deterministic mathematical programming,

$$
\left\{\begin{array}{l}
\min _{0}^{1}\left(\sum_{i=1}^{n} x_{i} \Phi_{i}^{-1}(\alpha)\right)^{2} \mathrm{~d} \alpha-\left(\int_{0}^{1} \sum_{i=1}^{n} x_{i} \Phi_{i}^{-1}(\alpha) \mathrm{d} \alpha\right)^{2} \\
\text { subject to: } \\
\int_{0}^{1} \sum_{i=1}^{n} x_{i} \Phi_{i}^{-1}(\alpha) \mathrm{d} \alpha \geq \delta \\
-\sum_{i=1}^{n} x_{i} \ln x_{i} \geq \gamma \\
x_{1}+x_{2}+\cdots+x_{n}=1 \\
x_{i} \geq 0, i=1,2, \cdots, n
\end{array}\right.
$$

and model(9) is converted into the following deterministic mathematical programming,

$$
\left\{\begin{array}{l}
\max -\sum_{i=1}^{n} x_{i} \ln x_{i} \\
\text { subject to: } \\
\int_{0}^{1} \sum_{i=1}^{n} x_{i} \Phi_{i}^{-1}(\alpha) \mathrm{d} \alpha \geq \delta \\
\int_{0}^{1}\left(\sum_{i=1}^{n} x_{i} \Phi_{i}^{-1}(\alpha)\right)^{2} \mathrm{~d} \alpha-\left(\int_{0}^{1} \sum_{i=1}^{n} x_{i} \Phi_{i}^{-1}(\alpha) \mathrm{d} \alpha\right)^{2} \leq \beta \\
x_{1}+x_{2}+\cdots+x_{n}=1 \\
x_{i} \geq 0, i=1,2, \cdots, n .
\end{array}\right.
$$


Example 4.1 Suppose that $\xi_{i} \sim \mathcal{N}\left(e_{i}, \sigma_{i}\right)$ are independent normal uncertain variables for $i=1,2, \cdots, n$. Then model (10), model (11) and model (12) respectively degenerate to the following deterministic programming models,

$$
\begin{aligned}
& \left\{\begin{array}{l}
\max \sum_{i=1}^{n} x_{i} e_{i} \\
\text { subject to: } \\
\sum_{i=1}^{n} x_{i} \sigma_{i} \leq \sqrt{\beta} \\
-\sum_{i=1}^{n} x_{i} \ln x_{i} \geq \gamma \\
x_{1}+x_{2}+\cdots+x_{n}=1 \\
x_{i} \geq 0, i=1,2, \cdots, n
\end{array}\right. \\
& \left\{\begin{array}{l}
\min _{i=1}^{n} \sum_{i} \sigma_{i} \\
\text { subject to: } \\
\sum_{i=1}^{n} x_{i} e_{i} \geq \delta \\
-\sum_{i=1}^{n} x_{i} \ln x_{i} \geq \gamma \\
x_{1}+x_{2}+\cdots+x_{n}=1 \\
x_{i} \geq 0, i=1,2, \cdots, n
\end{array}\right.
\end{aligned}
$$

and

$$
\left\{\begin{array}{l}
\max -\sum_{i=1}^{n} x_{i} \ln x_{i} \\
\text { subject to: } \\
\sum_{i=1}^{n} x_{i} e_{i} \geq \delta \\
\sum_{i=1}^{n} x_{i} \sigma_{i} \leq \sqrt{\beta} \\
x_{1}+x_{2}+\cdots+x_{n}=1 \\
x_{i} \geq 0, i=1,2, \cdots, n .
\end{array}\right.
$$

Proof. Note that $\xi_{i} \sim \mathcal{N}\left(e_{i}, \sigma_{i}\right)$ are independent normal uncertain variables for $i=1,2, \cdots, n$. It follows from Theorem 2.2 that $\sum_{i=1}^{n} x_{i} \xi_{i}$ has the inverse uncertainty distribution

$$
\Phi^{-1}(\alpha)=\sum_{i=1}^{n} x_{i}\left(e_{i}+\frac{\sqrt{3} \sigma_{i}}{\pi} \ln \frac{\alpha}{1-\alpha}\right),(0<\alpha<1) .
$$

Using the expected value formula and variance formula in Theorem 2.3 and Theorem 2.4, respectively, we have $E\left[\sum_{i=1}^{n} x_{i} \xi_{i}\right]=\sum_{i=1}^{n} x_{i} e_{i}$ and $V\left[\sum_{i=1}^{n} x_{i} \xi_{i}\right]=\left(\sum_{i=1}^{n} x_{i} \sigma_{i}\right)^{2}$. The proof is complete.
If the security returns are general uncertain variables, then it is difficult to obtain the uncertainty distributions of the portfolio returns. Therefore, we can use uncertain statistics to determine the uncertainty distribution and calculate its expected value and variance, respectively. Uncertain statistics first introduced by Liu [4] is a methodology for collecting and interpreting expert's experimental data by uncertainty theory. With the help of the designed questionnaire survey, the uncertainty distribution can be determined from expert's experimental data by the principle of least squares, the linear interpolation method, among others [4, 19].

\section{Numerical Examples}

In this section, the uncertain diversification models are applied to the data from Yan [14]. The data is composed of 5 security returns, which is shown in Table 1 . The returns of 5 securities are normal uncertain variables.

Table 1. Uncertain returns of 5 securities.

\begin{tabular}{llllll}
\hline Security $i$ & $\mathbf{1}$ & $\mathbf{2}$ & $\mathbf{3}$ & $\mathbf{4}$ & $\mathbf{5}$ \\
\hline Uncertain return & $\mathcal{N}(0,1)$ & $\mathcal{N}(1,2)$ & $\mathcal{N}(2,3)$ & $\mathcal{N}(3,4)$ & $\mathcal{N}(4,5)$ \\
\hline
\end{tabular}

Example 5.1 In order to compare the results of uncertain diversification model and mean-variance model (16) of Yan [14], we can use model (13) to search for an optimal portfolio.

$$
\left\{\begin{array}{l}
\max x_{2}+2 x_{3}+3 x_{4}+4 x_{5} \\
\text { subject to: } \\
x_{1}+2 x_{2}+3 x_{3}+4 x_{4}+5 x_{5} \leq 1.5 \\
x_{1}+x_{2}+x_{3}+x_{4}+x_{5}=1 \\
x_{i} \geq 0, i=1,2, \cdots, 5
\end{array}\right.
$$

When the bearable maximum risk $\beta$ and the minimum level measure of diversity $\gamma$ are set to $\beta=1.5$ and $\gamma=1$, respectively, model (13) becomes the following model (17),

$$
\left\{\begin{array}{l}
\max x_{2}+2 x_{3}+3 x_{4}+4 x_{5} \\
\text { subject to: } \\
x_{1}+2 x_{2}+3 x_{3}+4 x_{4}+5 x_{5} \leq 1.5 \\
-\sum_{i=1}^{5} x_{i} \ln x_{i} \geq 1 \\
x_{1}+x_{2}+x_{3}+x_{4}+x_{5}=1 \\
x_{i} \geq 0, i=1,2, \cdots, 5 .
\end{array}\right.
$$

We use LINGO to solve models (16) and (17) and the computational results are shown in Table 2 and Table 3, respectively. The two models obtain different optimal portfolios which have the same expected return 0.5 and the same bearable maximum risk $\beta=1.5$. However, the second portfolio has higher diversification than the first one, which is desired by the investor. 
Table 2. Optimal portfolios of model (16).

\begin{tabular}{llllll}
\hline $\mathbf{1}$ & $\mathbf{2}$ & $\mathbf{3}$ & $\mathbf{4}$ & $\mathbf{5}$ & $\beta$ \\
\hline $87.50 \%$ & $0.00 \%$ & $0.00 \%$ & $0.00 \%$ & $12.50 \%$ & 1.5 \\
\hline
\end{tabular}

Table 3. Optimal portfolios of model (17).

\begin{tabular}{lllllll}
\hline $\mathbf{1}$ & $\mathbf{2}$ & $\mathbf{3}$ & $\mathbf{4}$ & $\mathbf{5}$ & $\beta$ & $\gamma$ \\
\hline $65.93 \%$ & $22.67 \%$ & $7.80 \%$ & $2.68 \%$ & $0.92 \%$ & 1.5 & 1 \\
\hline
\end{tabular}

Example 5.2 Suppose that an investor wishes the level measure of diversity of his portfolio to be at least 1 , and the minimal expected return to be 2 . If he accepts variance as risk, then the uncertain diversification model (14) is equivalent to the following model (18),

$$
\left\{\begin{array}{l}
\min x_{1}+2 x_{2}+3 x_{3}+4 x_{4}+5 x_{5} \\
\text { subject to: } \\
x_{2}+2 x_{3}+3 x_{4}+4 x_{5} \geq 2 \\
-\sum_{i=1}^{5} x_{i} \ln x_{i} \geq 1 \\
x_{1}+x_{2}+x_{3}+x_{4}+x_{5}=1 \\
x_{i} \geq 0, i=1,2, \cdots, 5 .
\end{array}\right.
$$

Note that if we do not consider the diversity of the portfolio, then the model generates the model (19) in Yan [14].

$$
\left\{\begin{array}{l}
\min x_{1}+2 x_{2}+3 x_{3}+4 x_{4}+5 x_{5} \\
\text { subject to: } \\
x_{2}+2 x_{3}+3 x_{4}+4 x_{5} \geq 2 \\
x_{1}+x_{2}+x_{3}+x_{4}+x_{5}=1 \\
x_{i} \geq 0, i=1,2, \cdots, 5
\end{array}\right.
$$

With the help of LINGO, we find that model (18) and model (19) have the same minimum risk 3.00. In addition, under the expected return 2, we find that the minimum risk is still 3.00 when we decrease (increase) the preset entropy value $\gamma=0.5(1.5)$. In the other words, the results show that diversification using entropy has no effect on minimum risk. The results are given in Table 4 and Table 5.

Table 4. Results of model (18) with different preset entropy values.

\begin{tabular}{llllllll}
\hline$\gamma$ & $\mathbf{1}$ & $\mathbf{2}$ & $\mathbf{3}$ & $\mathbf{4}$ & $\mathbf{5}$ & $\beta$ & $\delta$ \\
\hline 0.5 & $4.21 \%$ & $11.33 \%$ & $69.74 \%$ & $9.69 \%$ & $5.03 \%$ & 3 & 2 \\
1 & $6.39 \%$ & $15.04 \%$ & $53.44 \%$ & $22.41 \%$ & $2.71 \%$ & 3 & 2 \\
1.5 & $14.41 \%$ & $16.47 \%$ & $37.42 \%$ & $18.13 \%$ & $13.57 \%$ & 3 & 2 \\
\hline
\end{tabular}

Table 5. Results of model (19).

\begin{tabular}{lllllll}
\hline $\mathbf{1}$ & $\mathbf{2}$ & $\mathbf{3}$ & $\mathbf{4}$ & $\mathbf{5}$ & $\beta$ & $\delta$ \\
\hline $50.00 \%$ & $0.00 \%$ & $0.00 \%$ & $0.00 \%$ & $50 \%$ & 3 & 2 \\
\hline
\end{tabular}

However, it can be seen that without the entropy constraint, the solution of the mean-variance model (18) is only concentrated on the security 1 and security 5 . With the entropy constraint, it is found from Table 4 that the greater the value of the preset entropy value is, the higher the portfolio diversification will be.

Example 5.3 Assume that the minimum expected return the investor can accept is 0.5 and the bearable maximum risk is 2.25. Based on the second variation of model (15), we obtain the following model (20),

$$
\left\{\begin{array}{l}
\max -\sum_{i=1}^{5} x_{i} \ln x_{i} \\
\text { subject to: } \\
x_{2}+2 x_{3}+3 x_{4}+4 x_{5} \geq 0.5 \\
x_{1}+2 x_{2}+3 x_{3}+4 x_{4}+5 x_{5} \leq 1.5 \\
x_{1}+x_{2}+x_{3}+x_{4}+x_{5}=1 \\
x_{i} \geq 0, i=1,2, \cdots, 5 .
\end{array}\right.
$$

In this model (20), we calculate the maximum proportion entropy to be 0.95 , i.e., the maximum level measure of diversity is 0.95 .

\section{Conclusions}

In this paper, the diversification problem of portfolio selection was discussed in an uncertain environment. An uncertain mean-variance-entropy diversification model was proposed which is an extension of Yan [14] uncertain meanvariance model. In our model, the mean, variance and proportion entropy were chosen as the objective function, risk measure and diversity measure, respectively. Next, a variation model was considered on the purpose of minimizing investment risk. Moreover, we used entropy as the objective function and discussed an uncertain diversification model via mean and variance constraints. With the help of the variance formula, the corresponding analytical mathematical models were deduced, which can be solved by the standard optimization problems. On the assumption that the security returns are all normal uncertain variables, some numerical examples were given. The results showed that the diversification models had higher diversification than the uncertain mean-variance model.

\section{Acknowledgements}

This work is supported by the National Natural Science Foundation of China Grant Nos. 61873108, 61703438 and 72071092 .

\section{References}

[1] A. Bera, S. Park, Optimal portfolio diversification using the maximum entropy principle, Econometric Reviews 27(2008) 484-512. 
[2] A. Golan, G. Judge, D. Miller, Maximum Entropy Econometrics RobustEstimation with Limited Data, Wiley, 1996.

[3] B. Liu, Uncertainty Theory, 2nd ed., Springer-Verlag, Berlin, 2007.

[4] B. Liu, Uncertainty Theory: A Branch of Mathematics for Modeling Human Uncertainty, Springer-Verlag, Berlin, 2011.

[5] B. Zhang, J. Peng, S. Li, Uncertain programming models for portfolio selection with uncertain returns, International Journal of Systems Science 46 (2015) 2510 2519 .

[6] C. Shannon, The Mathematical Theory of Communication, The University of Illinois Press, Urbana, 1949.

[7] D. Kahneman, A. Tversky, Prospect theory: an analysis of decision under risk, Econometrica 47 (1979) 263-292.

[8] H. Markowitz, Portfolio selection, Journal of Finance 7 (1952) 77-91.

[9] I. Usta, Y. Kantar, Mean variance skewness entropy measures: a multi-objective approach for portfolio selection, Entropy 13 (2011) 117-133.

[10] J. Chang, L. Sun, B. Zhang, J. Peng, Multi-period portfolio selection with mental accounts and realistic constraints based on uncertainty theory, Journal of Computational and Applied Mathematics 377 (2020) 112892.

[11] J. Kapur, H. Kesavan, Entropy Optimization Principles with Applications, Academic Press, New York, 1992.

[12] K. Yao, A formula to calculate the variance of uncertain variable, Soft Computing 19 (2015) 2947-2953.
[13] L. Chen, J. Peng, B. Zhang, I. Rosyida, Diversified models for portfolio selection based on uncertain semivariance, International Journal of Systems Science 48 (2017) 637-648.

[14] L. Yan, Optimal portfolio selection models with uncertain returns, Modern Applied Science 3 (2009) 76-81.

[15] P. Jana, T. Roy, S. Mazumder, Multi-objective Meanvariance-skewness model for portfolio optimization, Advanced Modeling and Optimization 9 (2007) 181-193.

[16] R. Zhou, R. Cai, G. Tong, Applications of entropy in finance: a review, Entropy 15 (2013) 4909-4931.

[17] S. Fang, J. Rajasekera, H. Tsao, Entropy Optimization and Mathematical Programming, Kluwer Academic, Boston, 1997.

[18] X. Huang, An entropy method for diversified fuzzy portfolio selection, International Journal of Fuzzy Systems 14 (2012) 160-165.

[19] X. Wang, Z. Gao, H. Guo, Delphi method for estimating uncertainty distributions, Information: An International Interdisciplinary Journal 15 (2012) 449-460.

[20] Y. Liu, M. Ha, Expected value of function of uncertain variables, Journal of Uncertain Systems 4 (2010) 181186.

[21] Y. Ning, H. Ke, Z. Fu, Triangular entropy of uncertain variables with application to portfolio selection, Soft Computing 19 (2015) 2203-2209.

[22] Y. Xia, B. Liu, S. Wang, K.K. Lai, A model for portfolio selection with order of expected returns, Computers and Operations Research 27 (2000) 409-422.

[23] Z. Peng, K. Iwamura, A sufficient and necessary condition of uncertainty distribution, Journal of Interdisciplinary Mathematics 13 (2010) 277-285. 